ORIGINAL ARTICLE

\title{
Evaluation of the role of a specialist tracheostomy service. From critical care to outreach and beyond
}

\author{
M G A Norwood, P Spiers, J Bailiss, R D Sayers
}

See end of article for

authors' affiliations

Postgrad Med J 2004;80:478-480. doi: 10.1136/pgmi.2003.016956

Correspondence to

MrM G A Norwood,

Department of Surgery,

Leicester General Hospital,

Leicester LE5 4PW, UK;

drmikenorwood@hotmail.

com

Submitted

10 November 2003

Accepted

20 December 2003

\begin{abstract}
The impact that a new specialist tracheostomy service, designed specifically for the care of patients with tracheostomies, was assessed in terms of type of tracheostomy tube used, time to first tube change, time to decannulation, and incidence of tracheostomy related complications in a teaching hospital with no on-site ear, nose, and throat facility. A total of 170 patients were studied. After service implementation, fewer patients $(17.6 \%, n=21)$ were discharged from the intensive treatment unit to the wards with tracheostomy tubes compared with the first group $(39 \%, n=20)(p=0.006)$, and the number of tracheostomy related complications on the wards were significantly reduced $(p=0.031)$.
\end{abstract}

$\mathrm{T}$ he use of tracheostomy tubes in critical care patients may be required for a number of different reasons. In the majority of cases, tracheostomy is performed as a temporary measure for patients requiring prolonged respiratory support and/or bronchial toilette. Tracheostomy tube placement can be performed via either a traditional open procedure or more commonly now by the percutaneous technique. Both techniques are generally regarded as "safe" procedures, ${ }^{1}$ with complication rates estimated at between $4 \%$ and $19 \% .^{2-4}$ Complications can be immediate, early and late, and include bleeding, incorrect placement with damage to neighbouring structures, pneumothorax, air leaks, tube blockage, and tube dislodgement. After the initial procedure, tube dislodgement and tube blockage are the most common and potentially most serious complications.

Since the development of percutaneous tracheostomy, there has been an increase in the number of hospitalised patients with tracheostomy tubes in situ, with a subsequent increase in the number of tracheostomised patients on general wards. ${ }^{56}$ The continued care of patients with tracheostomies requires a degree of specialist knowledge. These patients need regular input in the form of tube care, wound care, suction, education, advice, and prompt intervention in cases such as tube blockage and dislodgement. The ward staff also need to be fully aware of any potential complications. In situations such as general wards where this knowledge may be sparse due to rapid staff turnover, lack of senior support and the frequent use of bank staff, a structured support network for the ongoing care of the tracheostomised patient is required. It should be imperative that the perioperative and early care of patients with tracheostomies is carried out in a dedicated and structured manner, but literature to support such services is sparse. These issues have prompted the development of a specialist service within our hospital, designed to improve the quality of care delivered to patients with tracheostomy tubes, especially in patients discharged to the general medical and surgical wards.

Now that the specialist tracheostomy service has been established, we aim to assess the impact that this has had on patient care in terms of complication rates, the number of patients with tracheostomies, the duration of tracheostomy,

and the number of patients requiring nursing on the wards with tracheostomies.

\section{SPECIALIST TRACHEOSTOMY CARE AT LEICESTER GENERAL HOSPITAL}

Leicester General Hospital is one of three acute teaching hospitals in Leicester. These hospitals jointly serve a population of 960 000. Within Leicester General Hospital, there are eight intensive care (ITU) beds, four high dependency unit beds, four level 1 care beds, 83 acute surgical beds, and 175 acute medical beds. Other departments include urology, nephrology (including a renal high dependency unit), transplant surgery, cardiology (including a coronary care unit), medical rehabilitation, and elective orthopaedics. There is no on-site ear, nose, and throat service.

A new service, designed specifically for the care of patients with tracheostomy tubes, was implemented at Leicester General Hospital in April 1999. The service is composed of, and led by, a respiratory physiotherapist with a special interest in the care of patients with tracheostomies. An ITU outreach sister has also been recently appointed. This service is run separately to a more general "critical care outreach" that has been subsequently established. All patients receiving a tracheostomy are referred to the service on the same day as the tracheostomy procedure. They are subsequently followed up on a daily basis. An outreach service is also provided for all patients who are discharged from the critical care unit to the ward with a tracheostomy tube (including mini-tracheostomies) still present. These patients are also seen daily by the tracheostomy team, who are then responsible for continuing respiratory physiotherapy, airway suctioning, tube cleaning, tube changing, and decannulation. Other roles include education of the ward nursing staff in the ongoing care of patients with tracheostomy tubes. Out of hours support is provided by the critical care medical staff.

\section{SUBJECTS AND METHODS}

We used a case-control approach to assess the impact of this new service. Two groups of patients were recruited and compared. The first group (controls) were compiled from patients receiving a tracheostomy in the period before the development of the service, and the second group (cases) 
from patients with tracheostomy tubes placed after the service had been established. For simplicity, the cases will be referred to as the "post-service" group and the controls as the "pre-service" group.

For the pre-service group, data were collected retrospectively from the year before the establishment of the outreach (April 1998 to March 1999). During this time, no outreach facility existed. The name of all patients receiving a tracheostomy at Leicester General Hospital during this time were accessed via the medical records department using designated codes. A manual search of the patients' notes was then performed, with the relevant data being recorded.

For the post-service group, data were collected for two years (April 2001 to April 2003), after the implementation of the service, on all patients that had had placement of tracheostomy during this time. These data were collected prospectively on a daily basis from each patient seen by the outreach team, and recorded on a specifically designed proforma.

Data recorded included reason for tracheostomy, type of tracheostomy (for example, open, percutaneous, or minitracheostomy), date of first tube change, date of decannulation, and incidence of tracheostomy related complications.

\section{RESULTS}

A total of 60 patients were identified in the year before the tracheostomy specialist service (pre-service group). Of the 60 patients identified by the search, five sets of notes were unobtainable and four sets of notes had no record of a tracheostomy ever being performed, leaving a total of 51 patients to be included.

During the two years of the post-service group, there were a total of 111 tracheostomies performed, all of which were followed up daily until discharge or death. A further eight patients with tracheostomy tubes were transferred to Leicester General Hospital ITU from other hospitals, making up a total of 119 patients. All of these 119 patients are included in this study, as they were all seen daily by the outreach team.

\section{Type of tracheostomy}

In the pre-service group, $88 \%(n=45)$ of procedures used the percutaneous technique, and were performed on the ITU by trained anaesthetists. Seventy three percent $(n=37)$ of these were performed on postoperative general surgical patients, with the remaining $27 \%(\mathrm{n}=14)$ being performed on medical patients. Only $12 \%(n=6)$ of patients underwent an open procedure. All were a result of anatomical difficulties (for example, short, fat neck).

In the post-service group, $86.6 \%(n=103)$ of procedures were percutaneous. Eight of these were performed at another hospital, and the patient then transferred to Leicester General

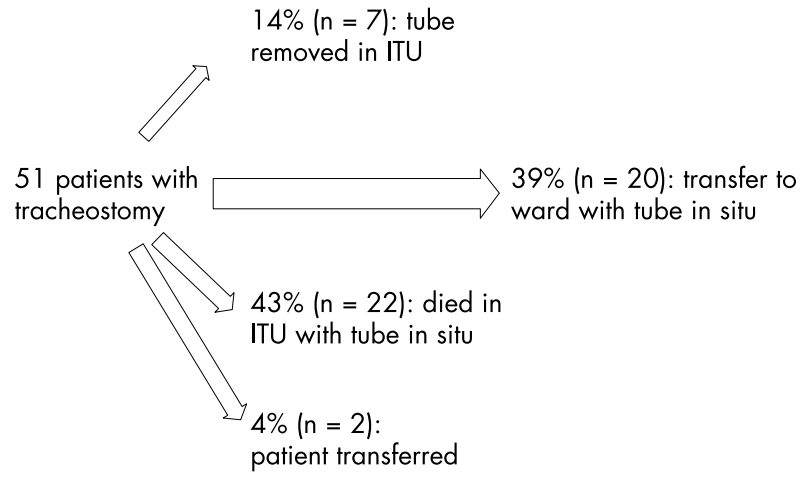

Figure 1 Outcome of patients after tracheostomy placement in ITU before implementation of the specialist tracheostomy service.

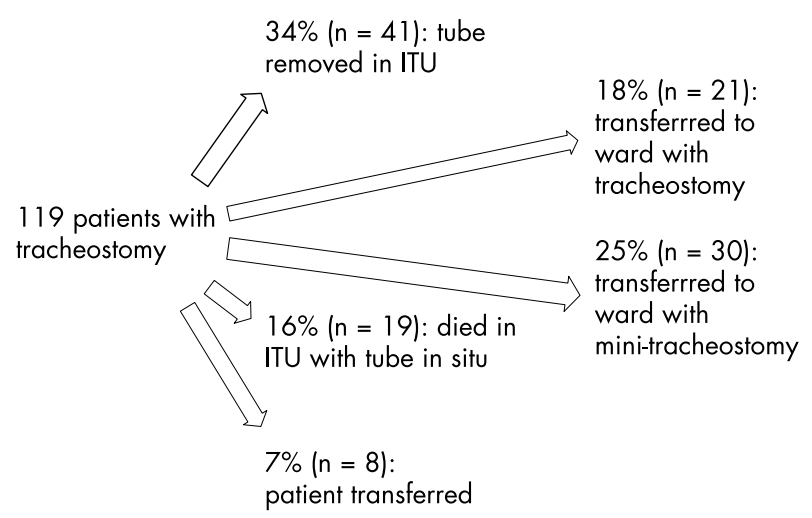

Figure 2 Outcome of patients after tracheostomy placement in the ITU after implementation of the specialist tracheostomy service.

Table 1 Number of tracheostomy related complications in both groups on the ITU

\begin{tabular}{lll}
\hline Type of complication & Pre-service & Post-service \\
\hline Patient decannulation & 0 & 2 \\
Blockage & 0 & 2 \\
Bleeding & 2 & 0 \\
Burst cuff & 0 & 1 \\
Total & 2 & 5 \\
\hline
\end{tabular}

\begin{tabular}{|c|c|c|}
\hline Type of complication & Pre-service & Post-service \\
\hline Blockage & $2^{*}$ & 0 \\
\hline Wound infection & 1 & 0 \\
\hline Tube dislodgement & 1 & 0 \\
\hline Total & 4 & 0 \\
\hline
\end{tabular}

Hospital. The remaining $13.4 \%(\mathrm{n}=16)$ of procedures were performed in theatre with an open technique, due to anatomical difficulties in 13 of the cases, and coagulopathy in the other three. Fifty five percent $(n=61)$ of tracheostomies performed at Leicester General Hospital were performed on surgical patients, with the remained being on either medical $(19 \%, n=21)$ or renal patients $(26 \%, n=29)$.

\section{Indications for tracheostomy}

In the pre-service group, the indications for tracheostomy were either for patients who were slow to wean from ventilation $(96 \%, \mathrm{n}=49)$, or for patients requiring regular bronchial suctioning $(4 \%, \mathrm{n}=2)$.

In the post-service group, all tracheostomies were performed on patients who were slow to wean from ventilation.

\section{Discharges to wards}

In the pre-service group, seven (14\%) patients had their tubes removed in the ITU, and $22(43 \%)$ patients died in the ITU with their tubes in situ. Two (4\%) patients were transferred out of the hospital and were lost to follow up, leaving a total of $20(39 \%)$ patients who were discharged from the ITU to the medical or surgical wards with their tracheostomy tube still in situ. In all of these 20 patients, the tube was a tracheostomy tube rather than a mini-tracheostomy tube (fig 1).

In the post-service group, 41 patients $(34 \%)$ were decannulated on the ITU, eight patients $(7 \%)$ were transferred to 
other hospitals, and 19 patients (16\%) died on the ITU before decannulation. Of the remaining 51 patients, $30(25 \%)$ were transferred from the ITU to wards with the mini-tracheostomy tubes in situ, leaving a total of only 21 patients (18\%) who were transferred from the ITU to the wards with a tracheostomy tube still in situ. This reduction in the number of patients transferred to the wards with tracheostomy tubes still in situ is statistically significant $(p=0.006$, Fisher's exact test) (fig 2).

\section{Complications of tracheostomy tubes}

Tracheostomy related complications are divided into those occurring on the ITU and those occurring on the wards. On the ITU there were two documented complications in the preservice group and five in the post-service group (table 1). On the wards, there were significantly fewer complications in the post-service group ( $p=0.031$, Fisher's exact test), with four documented complications in the pre-service group and zero complications in the post-service group (table 2). It is important to stress that of the four complications in the preservice group, there were two deaths as a direct result of tube blockage (denoted with ${ }^{*}$ in table 2 ). There were no tracheostomy related deaths in the post-service group.

\section{Other data}

In patients who survived, the mean time to decannulation was 18.96 days in the first group, and 18.5 days in the second group. The second group underwent their first tube change after an average of 11.5 days, but the first group did not have clear documentation of tube changes in the majority of cases, with only 10 patients undergoing a documented tube change (mean time to change was 16.2 days among documented cases).

\section{DISCUSSION}

The management and care of patients with tracheostomy tubes requires specialist knowledge. ${ }^{7}$ Despite statements that general ward nurses should be able to care for patients with tracheostomy tubes, ${ }^{8}$ recent research has shown a poor level of knowledge among ward nurses ${ }^{9}{ }^{10}$ leading to inevitable, potentially serious complications. ${ }^{5}$ Previous authors have discussed the potential benefits of tracheostomy specialist nurses, ${ }^{11}$ but published evidence of their existence and formal evaluation of existing services is sparse.

Within our hospital it had become apparent that patients with tracheostomy tubes on the general medical and surgical wards were still requiring regular input from ITU staff for support with their ongoing tracheostomy care after discharge from the ITU. There was also a general consensus that patients were having tracheostomy related complications which may have been prevented by a facility providing regular and specifically dedicated specialist input. This project has set out to assess the impact of this new service.

Although the number of patients in this study is small, it is one of the first to attempt evaluation of specialist services for patients with tracheostomy tubes, particularly of the role of outreach services for those patients on general medical and surgical wards.

The results from this study demonstrate a number of benefits. Firstly, and of most importance, there have been no serious incidents (for example, patient death as a direct result of a tracheostomy complication) since the implementation of the service. This is almost certainly due to the improvement of nurses' knowledge in the care of tracheostomised patients, and as a result of daily patient follow up by the outreach team, thus ensuring that patients are undergoing a minimum of at least daily tracheostomy care. Another important factor that is potentially responsible for the reduction in serious complications is the increased use of mini-tracheostomies. These are much safer to use than conventional tracheostomies, ${ }^{12}$ and there has been a large effort made by the tracheostomy team to ensure that patients leaving the ITU should have a mini-tracheostomy rather than a conventional tracheostomy wherever possible. As a result of the increased input that these patients are receiving since the development of this service, patients who are suitable for downsizing to a mini-tracheostomy have been better identified. Lastly, it should be realised that the actual number of complications in the first group in real terms are likely to be higher than the figure quoted. This is due to the retrospective data collection adopted in the first group, where an under-reporting of adverse events may have occurred, resulting in a lower than expected number of complications.

In addition to the reduction in the number of critical incidents, the outreach service has had further benefits. There has been an increase in the amount of nurse education in the care of patients with tracheostomies. This has improved patient care and has also reduced the burden on ITU staff. The outreach service as a result of daily follow up has also reduced the total length of time that tracheostomies are left in situ. It has also reduced the time to first and second tube changes, and finally it has increased the use of minitracheostomies as opposed to conventional tracheostomy, thus allowing patients to be more safely managed in the ward environment.

\section{CONCLUSION}

The implementation of a specialist service for the care of patients with tracheostomy tubes has significantly reduced the number of patients transferred from the ITU to the wards with tracheostomies in situ. The combination of fewer patients on the wards with tracheostomies, a daily tracheostomy outreach service, and better nurse education has resulted in significantly fewer tracheostomy related complications on the wards.

\section{Authors' affiliations}

M G A Norwood, University of Leicester Department of Surgery, Leicester General Hospital, Leicester, UK

P Spiers, J Bailiss, Leicester General Hospital, Leicester, UK R D Sayers, Leicester Royal Infirmary, Leicester, UK

This paper was presented as a poster at The European Society of Intensive Care Medicine on 6 October 2003 in Amsterdam.

\section{REFERENCES}

1 Cooper RM. Use and safety of percutaneous tracheostomy in intensive care. Report of a postal survey of ICU practice. Anaesthesia 1998;53:1209-12.

2 Cole IE. Elective percutaneous (Rapitrac) tracheostomy: results of a prospective trial. Laryngoscope 1994;104:1271-5.

3 Hill BB, Zweng TN, Maley RH, et al. Percutaneous dilatational tracheostomy: a report of 356 cases. J Trauma 1996;41:238-43.

4 Jeannon JP, Mathias D. Percutaneous (Portex) tracheostomy: an audit of the Newcastle experience. Ann R Coll Surg Engl 2000;82:137-40.

5 Heafield S, Rogers M, Karnik A. Tracheostomy management in ordinary wards. Hospital Medicine 1999;60:261-2.

6 Simpson TP, Day CJ, Jewkes CF, et al. The impact of percutaneous tracheostomy on intensive care unit practice and training. Anaesthesia 1999;54:186-9.

7 Woodrow P. Managing patients with a tracheostomy in acute care. Nursing Standard 2002;16:39-47

8 Audit Commission. Critical to success. The place of efficient and effective critical care services within the acute hospital. London: Audit Commission, 1999.

9 Day T, Farnell S, Haynes S, et al. Tracheal suctioning: an exploration of nurses' knowledge and competence in acute and high dependency ward areas. Journal of Advanced Nursing 2002;39:35-45.

10 Tanser SJ, Walker MB, Macnaughton PD. Tracheostomy care on the wardsan audit of nursing knowledge. Clinical Intensive Care 1997;8:105.

11 Russel C, Harkin H. The benefits of tracheostomy specialist nurses. Nursing Times 2001;97:40-1.

12 Wright CD. Minitracheostomy. Clin Chest Med 2003;24:431-5. 